\title{
Uso de produtos clorados na desinfecção de viveiros de piscicultura
}

\author{
Haluko Massago' e Bruno Corrêa da Silva²
}

\begin{abstract}
Resumo - Os peixes presentes nos viveiros podem ser predadores vorazes dos alevinos a serem povoados para o cultivo. Com o intuito de eliminar estes predadores, avaliou-se o hipoclorito de cálcio $65 \%\left(\mathrm{HC}_{65 \%}\right)$ e o dicloroisocianurato de sódio $65 \%$ $\left(\mathrm{DS}_{65 \%}\right)$ nos juvenis de tilápia, lambari e jundiá. Inicialmente, foram realizados testes em água clara para determinar concentração letal $(\mathrm{CL})$ para estas três espécies de peixe. Posteriormente, realizaram-se os testes em viveiro de piscicultura para alcançar a $\mathrm{CL}_{100}$. $\mathrm{A} \mathrm{CL}_{100}$ em 8 horas, nas águas claras foi de até $4 \mathrm{mg} \mathrm{L}^{-1} \mathrm{HC}_{65 \%}$ ou $6 \mathrm{mg} \mathrm{L}^{-1} \mathrm{DS}_{65 \%}$, para as três espécies. Já no viveiro, que apresentava 2,4\% de matéria orgânica no solo, a $\mathrm{CL}_{100}$ foi de $30 \mathrm{mg} \mathrm{L}^{-1}$ dos produtos teste. Pelo resultado, aconselha-se a concentração acima de $30 \mathrm{mg} \mathrm{L}^{-1}$ de cloro (3 $6 \mathrm{~g} \mathrm{~m}^{-2}$ de $\mathrm{HC}_{65 \%}$ ou $\mathrm{DS}_{65 \%}$ em viveiro com média de 10 20 $\mathrm{cm}$ de altura da água).
\end{abstract}

Termos para indexação: Cloro; Preparação do solo; Manejo.

\section{Use of chlorinated products in the disinfection of fish ponds}

Abstract - Different fishes present in farms can be voracious predators of fingerlings to be stocked in the rearing. To eliminate these predators, calcium hypochlorite $65 \%(\mathrm{HC} 65 \%)$ and sodium dichloroisocyanurate $65 \%$ (DS65\%) were evaluated in tilapia, yellow-tail characin and South America catfish juveniles. Initially, clear water assays were performed to determine the lethal concentration (LC) for these three fish species. Subsequently, tests were performed in a fish pond to observe the CL100. The CL100 at 8 hours in clear water was $4 \mathrm{mg} \mathrm{L}^{-1} \mathrm{HC} 65 \%$ or $6 \mathrm{mg} \mathrm{L}^{-1} \mathrm{DS} 65 \%$ for all three species. Already in ponds, containing $2.4 \%$ of organic matter in the soil, the CL100 was $30 \mathrm{mg} \mathrm{L}^{-1}$, for both products. According to the result, it advises the concentration above $30 \mathrm{mg}$ of chlorine $\mathrm{L}^{-1}$ (3-6 $\mathrm{g} \mathrm{m}^{-2}$ DS65\% or HC65\% in ponds containing an average of $10 \sim 20 \mathrm{~cm}$ water height).

Index terms: Chlorine; Fishpond preparation; Management.

O estado de Santa Catarina é o quinto estado brasileiro com maior produção de peixes em 2018, tendo produzido 45,7 mil toneladas (PeixeBR, 2019), com aproximadamente 30 mil produtores, que em sua maioria são pequenos agricultores com mão de obra familiar (SILVA et al., 2017). O principal sistema de cultivo utilizado em Santa Catarina na piscicultura é o cultivo em viveiros escavados. Neste sistema, um dos problemas é a presença de predadores de alevinos, entre eles, peixes de diversas espécies presentes no viveiro de povoamento que devem ser eliminados antes de iniciar o cultivo.

Para eliminar os patógenos e outros organismos indesejados normalmente aplica-se no solo dos viveiros cal virgem ou cal hidratada. A finalidade do óxido de cálcio, ou hidróxido de cálcio, é aumentar o pH do solo para eliminar os organismos causadores de doenças e seus vetores, sendo necessário pelo menos $1.000 \mathrm{~kg} \mathrm{ha}^{-1}$ de cal virgem (BOYD \& QUEIROZ, 2004). Os produtos à base de cloro podem ser de uso prático aos produtores devido à necessidade de menor quantidade, além de serem encontrados facilmente em lojas de produtos para piscinas. 0 cloro tem sido usado rotineiramente para desinfetar o abastecimento de água em criadouros de peixes e camarões, visto que compostos clorados atuam como agentes oxidantes fortes, eliminando os agentes patogênicos pelo contato (TONGUTHAl, 2000).

Para determinar necessidade do produto clorado para matar algumas espécies de peixes que podem aparecer em viveiros de piscicultura, foi realizado experimento com dois produtos clorados (hipoclorito de cálcio $65 \%-\mathrm{HC}_{65 \%}$, e dicloroisocianurato de sódio $65 \%$ $\left.\mathrm{DS}_{65 \%}\right)$ para as espécies lambari (Astyanax bimaculatus), tilápia (Oreochromis niloticus) e jundiá (Rhamdia quelen).

Inicialmente avaliou-se a concentração letal dos produtos para as três espécies em condições controladas. Foram utilizados lambaris com 2,8 $\pm 0,7 \mathrm{~g}$, tilápias com $3,6 \pm 1,7 \mathrm{~g}$ e jundiá com $69,9 \pm 22,2 \mathrm{~g}$. Para cada espécie e produto, realizaram-se os ensaios em 13 caixas de $80 \mathrm{~L}$ (20 peixes cada), sendo três controles e 10 concentrações do produto $(1,0 ; 1,5 ; 2,0 ; 2,5 ; 3,0 ; 4,0 ; 5,0 ; 6,0$; 8,0 e $\left.10,0 \mathrm{mg} \mathrm{L}^{-1}\right)$. Os parâmetros de qualidade de água antes do ensaio foram:

Recebido em: 7/3/2019. Aceito para publicação em 13/8/2019.

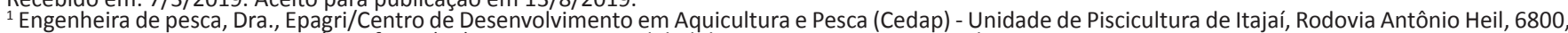

Bairro Itaipava, 88318-112 Itajaí, SC, fone: (47)3398-6353, e-mail: halukomassago@epagri.sc.gov.br.

${ }^{2}$ Engenheiro de aquicultura, Dr., Epagri/Cedap - Unidade de Piscicultura de Itajaí, fone: (47)3398-6324, e-mail: brunosilva@epagri.sc.gov.br. 
Tabela 1. Concentração letal (CL) do hipoclorito de cálcio 65\% $\left(\mathrm{HC}_{65 \%}\right)$ e dicloroisocianurato de sódio $65 \%\left(D_{65 \%}\right)$ para tilápia, lambari e jundiá em água clara

Table 1. Lethal concentration (LC) of calcium hypochlorite 65\% (HC65\%) and sodium dichloroisocyanurate 65\% (DS65\%) for tilapia, yellow-tail characin and South American catfish in clear water

\begin{tabular}{lllll}
\hline & & Tilápia & Lambari & Jundiá \\
\hline $\mathrm{HC}_{65 \%}\left(\mathrm{mg} \mathrm{L}^{-1}\right)$ & $\mathrm{CL}_{50}$ & 1,4 & 1,7 & 3,2 \\
& $\mathrm{CL}_{100}$ & 3,0 & 3,0 & 4,0 \\
\hline $\mathrm{DS}_{65 \%}\left(\mathrm{mg} \mathrm{L}^{-1}\right)$ & $\mathrm{CL}_{50}$ & 1,6 & 2,9 & 4,0 \\
& $\mathrm{CL}_{100}$ & 4,0 & 6,0 & 6,0 \\
\hline
\end{tabular}

temperatura $18^{\circ} \mathrm{C} ; \mathrm{pH} \mathrm{7,5}$; alcalinidade $28 \mathrm{mg} \mathrm{CaCO}_{3} \mathrm{~L}^{-1}$; amônia 0,17mg L"-1; nitrito $0,001 \mathrm{mg} \mathrm{L}^{-1}$; oxigênio dissolvido (OD) $>6,4 \mathrm{mg} \mathrm{L}^{-1}$ e cloro ativo $<0,03 \mathrm{mg}$ $\mathrm{L}^{-1}$. As mortalidades foram monitoradas durante 8 horas. Os dados foram submetidos à análise estatística por meio do método Trimmed Spearman-Karber, determinando a concentração letal mediana $\left(\mathrm{CL}_{50}\right)$ e concentração letal total $\left(\mathrm{CL}_{100}\right)$.

Nestes ensaios, após 8 horas de teste, as $\mathrm{CL}_{50}$ variaram de 1,4 a $3,2 \mathrm{mg} \mathrm{L}^{-1}$ $\mathrm{HC}_{65 \%}$, e 1,6 a $4,0 \mathrm{mg} \mathrm{L}^{-1} \mathrm{DS}_{65 \%}$. Já a $\mathrm{CL}_{100}$ variou de 3,0 a $4,0 \mathrm{mg} \mathrm{L}^{-1} \mathrm{HC}_{65 \%}$, e 4,0 a $6,0 \mathrm{mg} \mathrm{L}^{-1} \mathrm{DS}_{65 \%}$ (Tabela 1).

Nas condições testadas, $4 \mathrm{mg} \mathrm{L}^{-1}$ de $\mathrm{HC}_{65 \%}$ na água foi suficiente para eliminar juvenis de lambari, tilápia ou jundiá em menos de 8 horas. Para $\mathrm{DS}_{65 \%}$ a concentração letal foi mais elevada que $\mathrm{HC}$, devido à diferença de cloro ativo em cada produto ( $49,6 \%$ e $32,2 \%$ para $\mathrm{HC}_{65 \%}$ e $\mathrm{DS}_{65 \%}$, respectivamente). No geral a quantidade de cloro ativo necessária para eliminar $100 \%$ dos juvenis das três espécies avaliadas foi de $2 \mathrm{mg} \mathrm{L}^{-1}$.

Com base nos resultados, foi testado a $\mathrm{CL}_{100}$ (8 horas) em viveiros de $50 \mathrm{~m}^{2}$ com lâmina de água em média $20 \mathrm{~cm}$. Os parâmetros de qualidade de água dos viveiros na hora do ensaio apresentaram os seguintes valores: $30,7^{\sim} 32,0^{\circ} \mathrm{C}$; $\mathrm{pH} 7,1 \sim 8,1 ; \mathrm{OD}>5,7 \mathrm{mg} \mathrm{L}^{-1}$; alcalinidade 25,5 56,0mg CaCO $\mathrm{L}^{-1}$, e amônia 1,0mg $\mathrm{L}^{-1}$, cloro ativo $0,4 \mathrm{mg} \mathrm{L}^{-1}$. O solo apresentou pH 6,6 7,2 e matéria orgânica $1,4 \sim 2,4 \%$. Foram povoados com alevinos de tilápia e de lambari, em conjun- to, e foram avaliadas as concentrações de $5,10,20,25,30,35$, 40 e $45 \mathrm{mg} \mathrm{L}^{-1}$ do produto ( $\mathrm{HC}_{65 \%}$ ou $\mathrm{DS}_{65 \%}$ ), os quais foram diluídos em água e distribuídos em viveiro utilizando o regador. A avaliação foi qualitativa, considerando apenas as mortalidades totais ou parciais. Os viveiros onde foram adicionadas as concentrações de 5 a $25 \mathrm{mg} \mathrm{L}^{-1}$ dos produtos avaliados ( $\mathrm{HC}_{65 \%}$ ou $\mathrm{DS}_{65 \%}$ ) apresentaram mortalidades parciais para as duas espécies (tilápias e lambari), já com concentração acima de $30 \mathrm{mg} \mathrm{L}^{-1}$ apresentou mortalidade total para ambas. Estes valores estão dentro da faixa considerada necessária contra peixes, segundo Boyd \& Queiroz (2004). Após a obtenção do resultado, foi usado $30 \mathrm{mg} \mathrm{L}^{-1}\left(\mathrm{HC}_{65 \%}\right.$ ou $\mathrm{DS}_{65 \%}$ ) em seis viveiros pós-despesca de alevinagem da tilápia na unidade de piscicultura de Itajaí da Epagri, os quais eliminaram eficientemente os peixes. Vale ressaltar que esta concentração foi eficiente em viveiros com $2,4 \%$ de matéria orgânica. A atividade do produto clorado depende da concentração do cloro ativo presente, e sua eficiência é muito reduzida devido a fatores como a presença de matéria orgânica (CASTAGNOLLI, 1992; BOYD, 1979), luz (LE BRETON, 2009) e aumento do pH (BOYD, 2008). Em pH 7, 8 e 9, as necessidades de hipoclorito de cálcio $65 \%-\mathrm{HC}_{65 \%}$ para obter $1 \mathrm{mg} \mathrm{L}^{-1}$ de cloro livre são $1,54,4,71$ e $29,2 \mathrm{mg} \mathrm{L}^{-1}$, respectivamente (BOYD, 2008). Além disso, tanto o hipoclorito de cálcio, quanto o hipoclorito de sódio (HS) são desinfetantes contra bactérias e vírus, aplicáveis para água e estruturas da aquicultura (LE BRETON, 2009). Em ensaios agudos, Islam et al. (1997) verificaram a eficiência de HS contra bactéria Aeromonas salmonicida a $1,5 \mathrm{mg} \mathrm{L}^{-1}$ de cloro ativo durante 5 minutos, e Bicho et al. (2011) em peixezebra Danio rerio, $\mathrm{CL}_{100}$ (72 horas) de $10,1 \mathrm{mg} \mathrm{L}^{-1} \mathrm{HS} 10 \%$.

Tanto o hipoclorito de cálcio $65 \%$ quanto o dicloroisocianurato de sódio $65 \%$ foram eficientes para eliminar peixes avaliados em viveiros de piscicultura. Portanto, o uso para esta finalidade é interessante, devendo ser usadas concentrações acima de $30 \mathrm{mg} \mathrm{L}^{-1}$ destes produtos para a limpeza ictiológica.

\section{Agradecimentos}

À Fundação de Amparo à Pesquisa e Inovação do Estado de Santa Catarina (Fapesc), a Emidio Sant'Anna de Lara e a Silvio Demarch Filho.

\section{Referências}

ASSOCIAÇÃO BRASILEIRA DA PISCICULTURA (PeixeBR). Anuário peixeBR da piscicultura 2019: Produção brasileira cresce $4,5 \%$ e atinge 722.560 toneladas. São Paulo: Peixe BR, 2019. 146p. Disponível em: https://www. peixebr.com.br/Anuario2019/AnuarioPeixeBR2019.pdf. Acesso em: 04 Mai. 2020.

BICHO, R.C.; LADEWIG, J.C.L.; PITANGA, F.L.; MCDONOUGH, S.; OLIVEIRA, R.; SOARES, A.M.V.M.; NOGUEIRA, A.J.A.; DOMINGUES, I. Efeito do hipoclorito de sódio em diferentes níveis tróficos do ambiente aquático. CAPTAR: ciência e ambiente para todos, v.3, n.1, p.78-88, 2011. Disponível em: http:// revistas.ua.pt/index.php/captar/article/ view/2888/2705. Acesso em: 04 Mai. 2020.

BOYD, C.E. Water quality in warmwater fish ponds. Alabama: Crafitmaster Printers, Inc., 1979. 359p.

BOYD, C.E. Production sustainable aquaculture practices: Chlorine effective disinfectant in aquaculture. Global aquaculture advocate, p.52-53, 2008.

BOYD, C.E.; QUEIROZ, J.F. Manejo das condições de sedimento do fundo e da qualida-» 


\section{Jundiá}

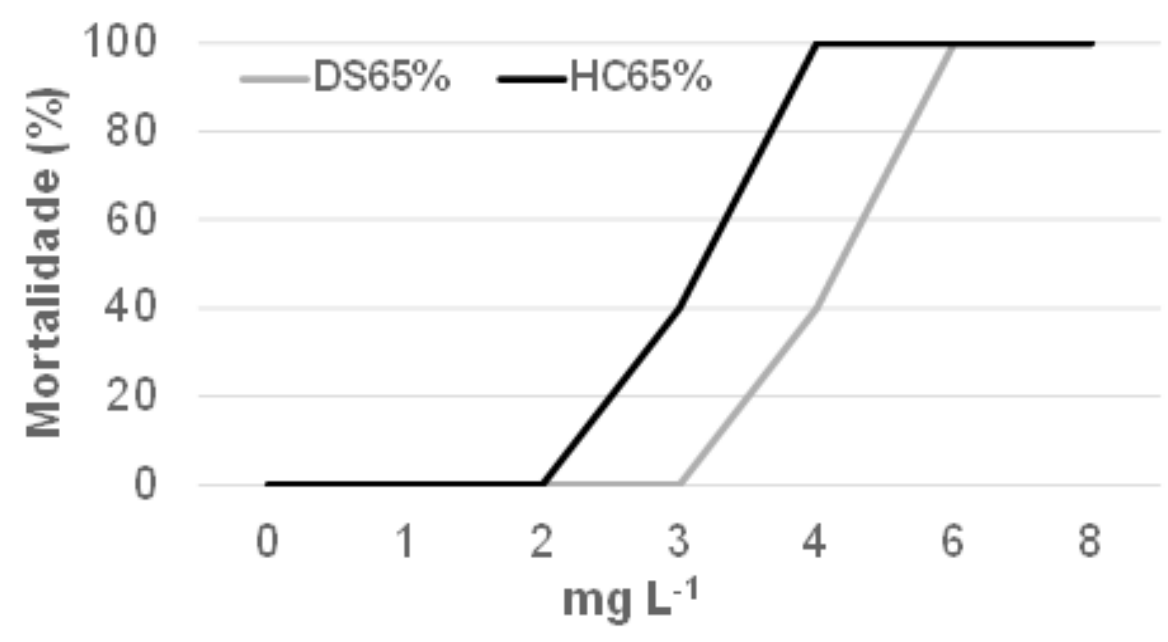

\section{Lambari}

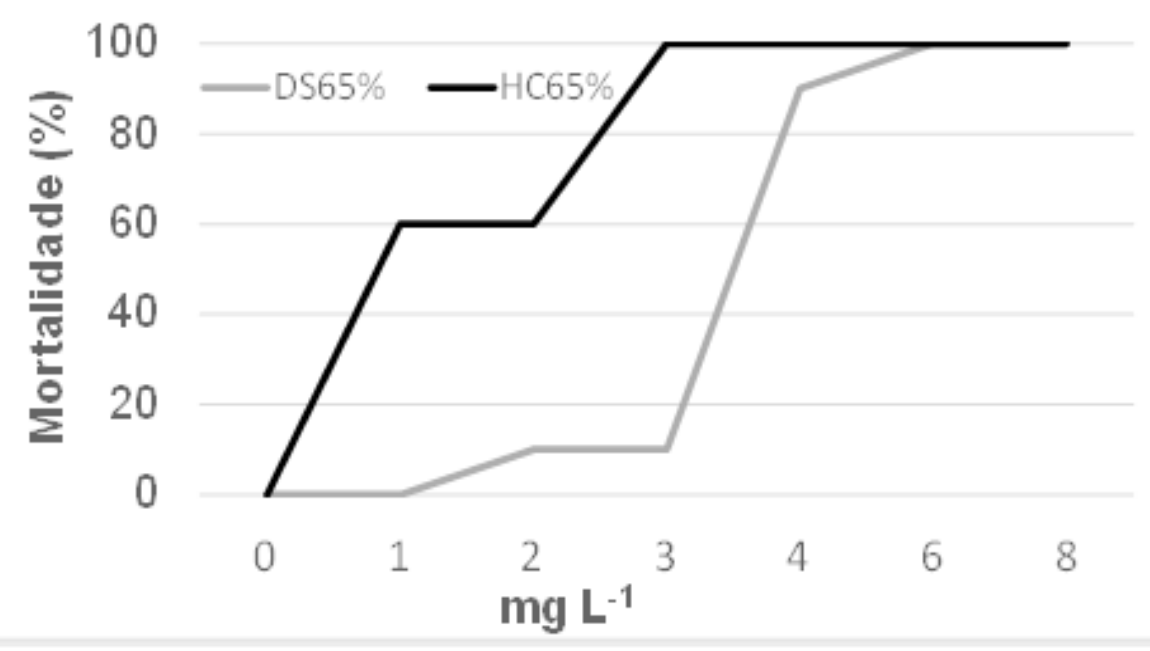

\section{Tilápia}

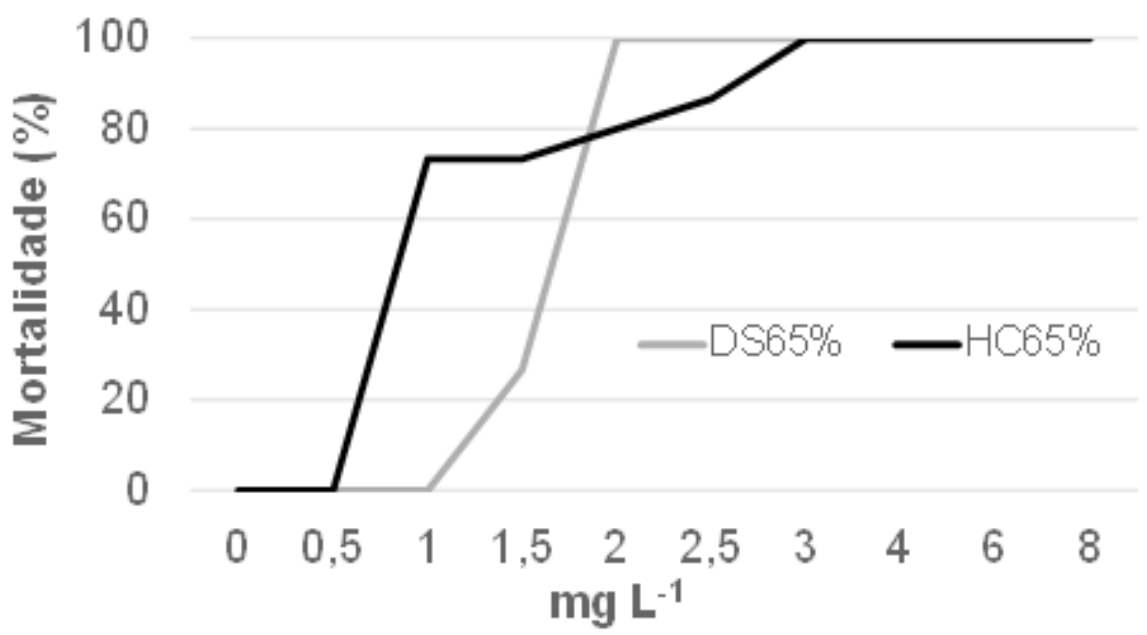

Figura 1. Curva de mortalidade da tilápia, lambari e jundiá para o hipoclorito de cálcio $65 \%$ $\left(\mathrm{HC}_{65 \%}\right)$ e dicloroisocianurato de sódio $65 \%\left(\mathrm{DS}_{65 \%}\right)$ em água clara

Figure 1. Mortality curve for tilapia, yellow-tail characin and South American catfish for calcium hypochlorite 65\% (HC65\%) and sodium dichloroisocyanurate 65\% (DS65\%) in clear water de da água e dos efluentes de viveiros. In: CYRINO, J.E.P.; URBINATI, E.C.; FRACALOSSI, D.M.; CASTAGNOLLI, N. (ed.). 2004. Tópicos especiais em piscicultura de água doce tropical intensiva. São Paulo: TecArt. p.25-43.

CASTAGNOLLI, N. Piscicultura de água doce. Jaboticabal: FUNEP, 1992. 189 p.

ISLAM, M.N.; FARUK, M.A.R.; INGLIS, V. Disinfection of aquarium effluents by chlorination and UV treatment. Bangladesh Journal of Fisheries Research, v.1, n.1, p.1-8, 1997. Disponível em: http://aquaticcommons. org/16352/1/BJFR1.1_001.pdf. Acesso em: 04 Mai. 2020.

LE BRETON A.D. Farming and health management: prevention and policy measures. In: ROGERS, C.; BASURCO, B. (ed.). The use of veterinary drugs and vaccines in Mediterranean aquaculture. Zaragoza: CIHEAM, 2009. p.207-220 (Options Méditerranéennes: Série A. Séminaires Méditerranéens; n. 86). Disponível em: https://om.ciheam.org/om/ pdf/a86/00801073.pdf. Acesso em: 04 Mai. 2020.

SILVA, B.C.; GIUSTINA, E.G.D.; MARCHIORI, N.C.; MASSAGO, H.; SILVA, F.M. Desempenho produtivo da piscicultura catarinense em 2015. Documentos no 268. Florianópolis: Epagri, 2017. 17p. ISSN 0100-8986. Disponível em: http://publicacoes.epagri.sc.gov.br/ index.php/RAC/article/view/176/102. Acesso em: 04 Mai. 2020.

TONGUTHAI, K. The use of chemicals in aquaculture in Thailand. In: Use of Chemicals in Aquaculture in Asia: Proceedings of the Meeting on the Use of Chemicals in Aquaculture in Asia 20-22 May 1996, Tigbauan, Iloilo, Philippines. Aquaculture Department, Southeast Asian Fisheries Development Center, 2000. p.207-220. Disponível em: http:// hdl.handle.net/10862/1815. Acesso em: 04 Mai. 2020.

TONGUTHAI, K. The use of chemicals in aquaculture in Thailand. In: Use of chemicals in aquaculture in Asia, 2000. Proceedings[...] Tigbauan: Filipinas: Aquaculture Department, Southeast Asian Fisheries Development Center, 2000. p.207-220. Disponível em: http://hdl.handle.net/10862/597. Acesso em: 04 Mai. 2020. 